\title{
The Reionization History in the Lognormal Model
}

\author{
Jiren Liu ${ }^{1}$, Li-Zhi Fang ${ }^{2}$, Long-Long Feng ${ }^{1,3}$, and Hong-Guang Bi ${ }^{2}$
}

Received __; accepted _

\footnotetext{
${ }^{1}$ Center for Astrophysics, University of Science and Technology of China, Hefei, Anhui 230026, China

${ }^{2}$ Department of Physics, University of Arizona, Tucson, AZ 85721

${ }^{3}$ National Astronomical Observatories, Chinese Academy of Science, Chao-Yang District, Beijing 100012, China
} 


\begin{abstract}
We study the evolution of baryonic gas before the reionization in the lognormal (LN) model of cosmic clustering. We show that the thermal history of the universe around the reionization can roughly be divided into three epochs: 1) cold dark age $z>z_{r}$, in which baryon gas is neutral, and opaque to Ly $\alpha$ photons; 2) hot dark age $z_{r}>z>z_{g p}$, in which a predominant part of baryon gas is ionized and hot, but it is still opaque to Ly $\alpha$ photons; 3) bright age $z<z_{g p}$, in which the universe is ionized highly enough to be transparent to Ly $\alpha$ photons. In the flat cold dark matter cosmological models given by WMAP and COBE, the difference of the two redshifts $z_{r}-z_{g p}$ is found to be as large as $\sim 10$ with $z_{r} \sim 17$ and $z_{g p} \sim 7$. This reionization history naturally yields a high optical depth to the $\mathrm{CMB} \tau_{e} \simeq 0.12-0.19$ observed by the TE polarization of the WMAP, and a low redshift $z_{g p}$ of the appearance of the Ly $\alpha$ Gunn-Peterson trough $z_{g p} \simeq 6-8$ in QSO's absorption spectra. The reason why the universe stays long in an ionized, yet Ly $\alpha$ opaque, stage is because the first photo-ionization heats the intergalactic gas effectively and has balanced the gravitational clustering a long period of time. Therefore, the result of a high $\tau_{e}$ and low $z_{g p}$ is a common feature of all the models considered. Besides the cosmological parameters, the only free parameter we used in the calculation is $N_{i o n}$, the mean ionization photons produced by each baryon in collapsed objects. We take it to be 40 - 80 in the calculation.
\end{abstract}

Subject headings: cosmology: theory - large-scale structure of the universe 


\section{Introduction}

The large scale structures of the universe evolved from initial mass density perturbations seeded by the quantum fluctuations of inflation. The power spectrum of the initial perturbation is of power law, $P(k) \propto k^{n}$, with index $n \sim 1$, and so the mass density perturbation is stronger on smaller scales. Accordingly, objects on smaller scales generally formed at earlier time. Small objects like primordial black holes could form at the early radiation-dominated era. Objects on scales of stars could form in the dark age, i.e. between the recombination and the reionization. These stars play the leading role of the history of the reionization.

The study of structure formation in the cosmic dark age is challenged recently by two observations. 1. The complete Ly $\alpha$ Gunn-Peterson trough in the absorption spectra of high redshift quasars shows that the reionization occurred probably at the redshift $z_{r} \simeq 6-8$ (Fan et al. 2002, and references therein). 2. The TE polarization of the Wilkinson Microwave Anisotropy Probe (WMAP) yields a model-independent value of the electron scattering optical depth to the cosmic microwave background (CMB), $\tau_{e}=0.17 \pm 0.04$, which requires the reionization redshift to be $z \simeq 11-30$ if the reionization happen rapidly (Kogut et al. 2003). Therefore, a problem naturally arisen is how to reconcile the two results, or why the reionization redshift from the Ly $\alpha$ spectrum is significantly lower than that given by the optical depth to the CMB. It has been realized that the high $\tau_{e}$ and low $z_{r}$ probably indicates that the reionization is not abrupt, but lasts a long period from $z=30-20$ to $6-8$ (Cen 2003; Haiman \& Holder 2003; Whyithe \& Loeb 2003; Holder et al. 2003; Hui \& Haiman 2003; Onken \& Miralda-Escudé 2003). The further questions we might ask are how to describe the long period of the reionization and which is the physical mechanism leading to such a long period of the reionization? This paper is to address these problems using a semi-analytical approach in the lognormal (LN) model. 
The LN model assumes that the mass field of intergalactic medium (IGM) is given by an exponential mapping from the linear field (Bi 1993; Bi \& Davidsen 1997). The probability distribution function (PDF) of the IGM field is then lognormal. The first argument in supporting the lognormal PDF is probably from the isothermal model of the IGM in the gravitational potential as

$$
\rho(\mathbf{x}) \propto \exp \left[-\frac{\mu m_{p}}{\gamma k T} \phi_{d m}(\mathbf{x})\right] .
$$

where $\rho(\mathbf{x})$ is the IGM mass density and $\phi_{d m}(\mathbf{x})$ the gravitational potential. $T$ and $\mu$ are, respectively, the temperature and molecular weight of the gas, and $\gamma$ is the polytropic index. Eq. (1) is the well-known IGM model of intracluster gas (Sarazin \& Bahcall 1977). Zeldovich et al. (1990) pointed that when the gravity potential $\phi_{d m}(\mathbf{x})$ is a random field with a Gaussian PDF, the field $\rho(\mathbf{x})$ of eq.(1) is lognormal. It has been shown that the lognormal solution is a reasonable approximation of the dynamical equations of the IGM (e.g. Jones 1999; Matarrese \& Mohayaee 2002). Therefore, the LN is dynamically legitimate to model the weakly nonlinear properties (Bi \& Davidsen 1997), and highly non-Gaussian features (Feng \& Fang 2000) of the IGM. Recently it is also used to study the collapsing of hydrogen clouds of the IGM (Bi et al. 2003, here after BFFJ).

As has been emphasized in BFFJ, the formation of the first generation of collapsed hydrogen clouds is given by rare events, which is sensitive to the tail of the mass field PDF on the high perturbation side. It is noted that the LN PDF is of long tailed and predicts a higher probability of rare events of high perturbation than PDFs without a long tail. Upon this consideration, the BFFJ showed that the reionization lasts a period from redshift 15 to about 10. However, the BFFJ did not considered the reaction of the evolved IGM on the reionization. During the reionization, the IGM underwent a dramatic evolution from neutral to ionized hydrogen, from cold to hot gas, and from low to high entropy states. Consequently, the clustering behavior of the IGM should also undergo a remarkable 
evolution in the epoch around the reionization. On the other hand, the clustering of the underlying dark matter is not affected by the reionization. Therefore, we must analyze the impact of the IGM evolution upon the reionization history.

The LN model directly provides the spatial distributions of the IGM. It is effective to reveal the reaction of the evolution of the IGM on the reionization. We will show that a long period of the recombination is a natural outcome of the LN model if considering the evolution of the IGM at the reionization. A long period of the reionization history generally yield a high optical depth $\tau_{e}$ to the CMB and a low reionization redshift to the Ly $\alpha$ forests. We will calculate these quantities in the cold dark matter cosmological models.

The paper is outlined as follow. In $\S 2$ we will describe the LN model of the reionization. The history of hydrogen reionization will be discussed in $\S 3$. Finally $\S 4$ gives the discussion and conclusion.

\section{The Lognormal Model}

\subsection{Mass Field of Baryonic Gas}

In the LN model, the mass field of the IGM is given by an exponential mapping of the linear density field of the underlying dark matter as

$$
\rho(\mathbf{x})=\bar{\rho} \exp \left[\delta_{0}(\mathbf{x})-\sigma_{0}^{2} / 2\right]
$$

where $\sigma_{0}$ is the variance of the linear Gaussian field of the dark matter filtered on scales of the Jeans length $\lambda_{b}$ of the IGM; $\delta_{0}(\mathbf{x})$ is the linear density contrast of dark matter $\delta_{d m}(\mathbf{x})$ smoothed by a window on the Jeans scale, i.e. its Fourier counterpart is (Bi \& Davidsen 1997)

$$
\delta_{0}(\mathbf{k})=\frac{\delta_{d m}(\mathbf{k})}{1+x_{b}^{2} k^{2}}
$$


where $x_{b}$ is $1 / 2 \pi$ of the comoving Jeans length of the IGM.

Obviously, when the fluctuation $\delta_{0}(\mathbf{x})$ is small, i.e. $\delta_{0}(\mathbf{x}) \ll 1$, eq. (2) reduces to approximately $\delta(\mathbf{x}) \simeq \delta_{0}(\mathbf{x})$, where $\delta(\mathbf{x})=[\rho(\mathbf{x})-\bar{\rho}] / \bar{\rho}$ is the density contrast of the IGM. Moreover, when the fluctuation $\delta_{0}(\mathbf{x})$ is large, combining eqs.(2) and (3) yields eq.(1) (Bi \& Davidsen 1997). Since the initial density perturbation is stronger on smaller scales, the perturbations of the dark matter gravitational potential $\phi_{d m}(\mathbf{x})$ are larger on smaller scales. Thus, even at early universe the IGM field on small scales may already be in the state described by eq.(1). Thus, it would be not appropriate to assume that the IGM field is always Gaussian on all scales. The LN model provides a uniform description of the IGM distribution in the linear, weakly nonlinear and lognormal regime at various scales and redshifts.

Since the random field $\delta_{0}(\mathbf{x})$ is Gaussian, the PDF of the field $\rho(\mathbf{x})$ is lognormal as

$$
p(\rho / \bar{\rho})=\frac{1}{(\rho / \bar{\rho}) \sigma_{0} \sqrt{2 \pi}} \exp \left[-\frac{1}{2}\left(\frac{\ln (\rho / \bar{\rho})+\sigma_{0}^{2} / 2}{\sigma_{0}}\right)^{2}\right], \quad \rho \geq 0 .
$$

which gives the probability density of the event such that the mass density within an area on scales of the Jeans length is found to be equal to $\sim \rho / \bar{\rho}$. The tail of the PDF eq.(4) is $\sim \exp \left[-(1 / 2)(\ln \rho / \bar{\rho})^{2} / \sigma_{0}^{2}+\ln \rho / \bar{\rho}\right]$. Therefore, the probability of $\rho / \bar{\rho}$ for a given $\sigma_{0}$ would be equal to the probability of $(\rho / \bar{\rho})^{2}$ for $2 \sigma_{0}$. Namely, the change of variance $\sigma_{0}$ by a factor 2 leads to a change of the possible high density events from $\rho / \bar{\rho}$ to $(\rho / \bar{\rho})^{2}$.

Obviously, the PDF is normalized as $\int_{0}^{\infty} p(x) d x=1$, and the variance of $\rho / \bar{\rho}$ is given by (Vanmarcke 1983)

$$
\sigma=\left[e^{\sigma_{0}^{2}}-1\right]^{1 / 2}
$$

The $n^{\text {th }}$ moment of $\rho$ is

$$
\overline{(\rho / \bar{\rho})^{n}}=\exp \left[\left(n^{2}-n\right) \frac{\sigma_{0}^{2}}{2}\right]
$$

Equation (6) shows $\left[\overline{\left(\rho / \bar{\rho}^{n}\right.}\right]^{1 / n}>\left[\overline{(\rho / \bar{\rho})^{2}}\right]^{1 / 2}$. That is, the ratio between the high- and 
low-order moments is divergent in the limit of $n \rightarrow \infty$. A field with such a divergent moment ration, by definition, is intermittent (Zel'dovich, Ruzmaikin, \& Sokoloff 1990). Actually, LN is typically a mathematical model of intermittent field. This property is in good consistent with the detected intermittency of the transmitted flux of QSO Ly $\alpha$ absorption (Jamkhedkar, Zhan \& Fang 2000; Feng, Pando \& Fang 2003, Jamkhedkar et al. 2003).

\subsection{Jeans Length of Baryonic Matter}

Equation (2) shows that, in the LN model, the clustering features of the baryonic gas is characterized by the variance $\sigma_{0}$ of the linear density field on the scale of the Jeans length. Primordial baryons, created at the time of nucleosynthesis, recombines with electrons to become neutral gas at $z \sim 1000$. Thereafter, the gas cools down adiabatically with the expansion of the universe. In a homogeneous universe with the mean mass density $\rho_{m}$, the Jean length of gaseous baryonic matter or the IGM is defined by $\lambda_{b} \equiv v_{s}\left(\pi / G \rho_{m}\right)^{1 / 2}$, where $v_{s}$ is the sound speed of gas. The corresponding Jeans mass is $m_{J}=(4 \pi / 3) \lambda_{b}^{3} \rho_{m}$. In a comoving scale of $x_{b}=\lambda_{b} / 2 \pi$, we have (Bi \& Davidsen 1997)

$$
x_{b}=\frac{1}{H_{0}}\left[\frac{2 \gamma k_{B} T}{3 \mu m_{p} \Omega_{m}(1+z)}\right]^{1 / 2} .
$$

The polytropic index is assumed to be $\gamma=5 / 3$, and hydrogen temperature thus follows $T \propto \bar{\rho}^{2 / 3}$, where $\bar{\rho}$ is the mean mass density of baryonic matter. At $z=15$, the hydrogen temperature is $\sim 4 \mathrm{~K}$ (Medvigy \& Loeb 2001), and the Jeans length $x_{b} \sim 1.2 \mathrm{kpc}$. The redshift evolution of the comoving Jeans length $x_{b}$ is approximately given by $(1+z)^{1 / 2}$.

During the reionization, the baryonic gas will be heated by the UV ionizing background from few degrees of temperature to $\sim 1.3 \times 10^{4} \mathrm{~K}$; and correspondingly, there is about 2 magnitudes increasing in $x_{b}$ or 6 magnitudes in the Jeans mass (e.g. Ostriker \& Gnedin 
1996). After the reionization, the IGM temperature is maintained at about $\sim 10^{4} \mathrm{~K}$ by the UV background photons, and therefore, $x_{b}$ will gradually increase with the decrease of $z$ due to the factor $(1+z)$ in eq. $(7)$.

For the variance $\sigma_{0}$ in eq.(2), we consider three fiducial cosmological models. Two are inferred from the WMAP, and one from the COBE. The cosmological parameters of these models are listed in Table 1. We use the linear power spectrum of the dark matter given by Eisenstein \& Hu (1999). The linear variance is approximately growing with redshift as $\sigma_{0}(z) \propto 1 /(1+z)$. For cosmological models of $\Omega=1$ and $\Omega_{\Lambda} \neq 0$, the linear growth of density perturbations is not exactly following the factor $1 /(1+z)$ (Lahav et al. 1991). We take account of this effect by replacing the factor $1 /(1+z)$ by $1 / g\left(\Omega_{m}, \Omega_{\Lambda}\right)(1+z)$, where the $g$ factor is

$$
g\left(\Omega_{m}, \Omega_{\Lambda}\right)=\frac{2.5 \Omega_{m}}{\Omega_{m}^{4 / 7}-\Omega_{\Lambda}+\left(1+\Omega_{m} / 2\right)\left(1+\Omega_{\Lambda} / 70\right)} .
$$

This correction is approximately reasonable at $z>1$ (Avelino \& de Carvalho 1999, Carroll et at. 1992). For the models of WMAP1, WMAP2 and COBE, the $g$ factor is also shown in Table 1.

Because the powers of the linear perturbations $k^{3} P(k)$ in all of these models are smaller on larger scales, the two order magnitude jump of $x_{b}$ at the reionization generally leads to a drop-off of the variance $\sigma_{0}$. A typical curve of $\sigma_{0}$ for the model WMAP2 is displayed in Fig. 1 , in which the reionization is assumed to happen instantly around redshift $z_{r}=17$. The dotted line in Fig. 1 is for $\sigma_{0}$ in case of no reionization occurring. The zigzag $z$-dependence around the reionization is a common feature of the $\sigma_{0}$ curve. For instance, the Fig. 2 of the BFFJ shows also a zigzag at $z=7$. In the BFFJ, it is assumed that the reionization takes place at redshift $z_{r}=7$. In this paper, we will not use a priori zigzag redshift $z_{r}$, but determine it by a self-consistent calculation. It should be pointed out that the zigzag $z$-dependence of $\sigma_{0}$ does not affect the evolution of dark matter, of which the clustering is 
Table 1: Optical depth in three cosmological models

\begin{tabular}{llllllll}
\hline model & $\Omega_{m}$ & $\Omega_{\Lambda}$ & $\Omega_{b}$ & $n_{s}$ & $\sigma_{8}$ & $h$ & $g$ \\
\hline WMAP1 & 0.27 & 0.73 & 0.044 & 0.97 & 0.84 & 0.71 & 0.758 \\
WMAP2 & 0.29 & 0.71 & 0.047 & 0.99 & 0.9 & 0.72 & 0.772 \\
COBE & 0.3 & 0.7 & 0.045 & 1 & 0.85 & 0.7 & 0.797 \\
\hline
\end{tabular}

basically regardless the reionization.

\subsection{Cumulative Mass Fraction and Clumping Factor}

For a field with the PDF of eq.(4), the cumulative mass fraction $M(>\rho / \bar{\rho})$, i.e. the fraction of mass in regions having mass density larger than a given $\rho / \bar{\rho}$, is

$$
M[>(\rho / \bar{\rho})]=\int_{\rho / \bar{\rho}}^{\infty} x p(x) d x=\frac{1}{2} \operatorname{erfc}\left[\frac{\ln (\rho / \bar{\rho})}{\sqrt{2} \sigma_{0}}-\frac{\sigma_{0}}{2 \sqrt{2}}\right] .
$$

which implies that $M[>(\rho / \bar{\rho})]$ is a strongly varying function of the variance $\sigma_{0}$, but weak for the variable $\rho / \bar{\rho}$. Fig. 2 demonstrates a cumulative mass fraction for $\rho / \bar{\rho}=2,6$ and 10, in which $\sigma_{0}$ is taken from Fig. 1, and using the dotted line for $z<z_{r}$, i.e. without considering the drop-off of $\sigma_{0}$ at $z_{r}$. Thus, Fig. 2 can be only used at redshift $z>z_{r}$.

The decrease of $M(>\rho / \bar{\rho})$ with $\rho / \bar{\rho}$ is much slower than a Gaussian PDF, or PDFs with a Gauss-like tail. For a Gaussian tail, we have $M(>10) / M(>6) \simeq \exp \left[\left(-10^{2}+6^{2}\right) / 2 \sigma_{0}^{2}\right]$, or $M(>10) / M(>6)<\exp (-30)$, when $\sigma_{0} \leq 1$. However, Fig. 2 shows that even when $z$ is as high as $20, M(>10)$ is less than $M(>6)$ only by a factor less than 10 . This is obviously due to the long tail behavior of the lognormal PDF, which gives a larger probability of high density events at high redshifts when the variance $\sigma_{0}$ is still small.

The variable $\rho / \bar{\rho}$ in eq. (9) is the density within an area on scales of the Jeans length, and therefore, the area with $\rho / \bar{\rho}>1$ has mass larger than the Jeans mass. However, these 
regions are not always in collapsing. This is because that the IGM clouds have no time to collapse even when the cloud mass is larger than the Jeans mass. BFFJ found that at redshift $z \simeq 7$, only clouds with density $\rho / \bar{\rho}>6$ are in the turning phase. The threshold $\rho / \bar{\rho} \simeq 6$ is much larger than the Jeans mass. In other words, the clustering on the Jeans length scales is significantly suppressed. The suppression of collapsing on the Jeans length scales has also been noted in studying the so-called filtering scale (Gnedin \& Hui 1998).

The threshold for the clouds going to collapsing at redshift $z>7$ is a little lower than 6. Therefore, the mass fraction of collapsed clouds can be estimated by the cumulative mass fraction $M(>6)$. One can estimated the velocity dispersion of the collapsed clouds by considering that in the LN model the collapsed clouds in potential well is probably isothermal [eq.(1)]. In this case, the velocity dispersion of the collapsed clouds is approximately (e.g. Jing \& Fang 1994)

$$
\sigma_{v} \simeq 13 H_{0} x_{b}(1+z)^{1 / 2} \Omega_{m}^{1 / 2}(\rho / \bar{\rho})^{1 / 2}
$$

Thus, for $h=0.7, \Omega_{m}=0.3$, the velocity dispersion of the collapsed clouds of $\rho / \bar{\rho}>6$ is from $>3.8 \mathrm{~km} \mathrm{~s}^{-1}$ at $z=10$ to $>7.7 \mathrm{~km} \mathrm{~s}^{-1}$ at $z=20$. If using $\rho / \bar{\rho}>10$, we have $\sigma_{v}>5.0$ $\mathrm{km} \mathrm{s}^{-1}$ at $z=10$ to $>9.8 \mathrm{~km} \mathrm{~s}^{-1}$ at $z=20$. Therefore, all these clouds have mass larger than $10^{4} \mathrm{M}_{\odot}$, the $H_{2}$ cooling can take place.

The so-called clumping factor is the second moment of the IGM density field defined by

$$
C(z) \equiv \overline{(\rho / \bar{\rho})^{2}}=e^{\sigma_{0}^{2}(z)}
$$

Fig. 3 plots the clumping factor $C(z)$ in the three models with $\sigma_{0}$ without considering the drop-off at $z_{r}$. It can only be used for redshift $z>z_{r}$. The clumping factor is significantly $z$-dependent. For all three models, $C(z)$ are large only at redshift $z<10$, and rapidly decreases to $<3$ at redshifts $z>15$. At a first glance, it seems to be a contradict between a higher probability of clustering and a lower clumping factor. Actually, it is nothing but 
a typical feature of intermitted field, which consists of high density spikes and low density regions between the spikes, and therefore, dense objects can be effectively formed in the spikes, while the mean clumping factor over the whole space is relatively low.

\section{History of the Reionization}

\subsection{Clustering of Baryonic Gas around Reionization}

From Fig. 1, one can see that the $z$-dependence of $\sigma_{0}$ before and after the reionization $z_{r}$ are similar. Before $z_{r}, \sigma_{0}$ is increased from 0.4 to 0.8 , and after $z_{r}, \sigma_{0}$ is again to increase from about 0.4 to higher values. A fall of $\sigma_{0}$ is by a factor of $\sim 2$ at $z_{r}$. Of course, the change of $\sigma_{0}$ at the drop is dependent on $z_{r}$. In this section, we only qualitatively describe the effect of the $\sigma_{0}$ drop on the clustering of baryonic gas. Quantitative calculation will be given in next two subsections.

As emphasized in $§ 2.1$, the cumulative mass fraction is a strong function of $\sigma_{0}$. The drop of $\sigma_{0}$ by a factor of two leads to a substantial change of the cumulative mass fraction.

One can then divide the IGM clustering into two phases, before and after redshift $z_{r}$. Before the reionization, structure formation was weak when the variance $\sim 0.4$, and became more active when the variance reached $\sim 0.8$. Just after $\sigma_{0}$ dropping, the structure formation tended to inactive again as the variance reduced to $\sim 0.4$. Soon after the variance growing up to $\sim 0.8$, the structure formation was built up once more. Therefore, there are two phases, in which the rate of structure formations are high. The early phase is just before $z_{r}$, and the late phase is at the second time of $\sigma_{0} \geq 0.8$.

The physical reason for the two-phase scenario of structure formation is obvious. Baryonic gas falls into dark matter gravity wells and the first generation of stars forms before $z_{r}$. Because the UV ionization photons background is gradually established from the 
first generation stars, baryonic gas is heated to temperature $10^{4} \mathrm{~K}$, and the Jeans length becomes bigger. The irregularities or clustering within the big Jeans scale would be smeared out by thermal motion. Since the density fluctuations on the big Jeans scales would still be in the linear regime, the process of the structure formation is significantly suppressed even halted. At the end of this smearing out, some ionized hydrogen will move away from the bottom of the gravity wells of dark matter halos. Eq.(1) also shows that when the temperature $T$ increases by a factor of $10^{4}$, many nonlinear structures will disappear. The clustering will recover till the variance $\sigma_{0}$ growing again. In the second phase, the objects form from ionized IGM, and their masses range in the order of $10^{9-10} M_{\odot}$.

In the two-phase scenario, the structure formation will be slowed down just after the reionization. Consequently, there is no enough UV photons produced by stars to maintain a fully ionized IGM, and a significant fraction of HI remains in the IGM. In this case, the IGM is opaque for Ly $\alpha$ photons. The IGM becomes transparent for Ly $\alpha$ photons till the redshift at which the rate of star formation grows again to be able to produce enough UV photons for maintaining a highly ionized IGM. Therefore, we can define two redshifts: (1) $z_{r}$, the redshift of reionization, which is the first time when the IGM was fully (or almost fully) ionized and heated up to temperature $\sim 10^{4} \mathrm{~K} ;(2) z_{g p}$, the redshift of the Gunn-Peterson transparency, which is the starting time for IGM entering into the state such that the volume-averaged fraction of $\mathrm{HI}$ is less than $10^{-4}$, and the Gunn-Peterson absorption ceased. In a word, the clustering of the IGM is suppressed during the period from $z_{r}$ to $z_{g p}$, and thus leads to the reionization epoch lasting a long period from $z_{r}$ to $z_{g p}$.

The two-phase scenario is based on the $z-\sigma_{0}$ relation indicated in Fig. 1. Obviously, this $z$-dependence of $\sigma_{0}$ is approximate, as we assumed that the temperature of the IGM suddenly increased by a factor of $10^{4}$. More precisely, the sharp decline of $\sigma_{0}$ at $z_{r}$ should be replaced by a soften transition from the top branch to the bottom branch in the $z-\sigma_{0}$ 
plane. Nevertheless, the mechanism for a long period reionization outlined above still works, as it essentially depends only on the decrease of $\sigma_{0}$ with the heating and ionizing IGM.

\subsection{The Redshift of Reionization}

Let us first calculate the reionization redshifts $z_{r}$. The reionization of hydrogen clouds is usually characterized by the filling factor of ionized hydrogen $Q_{H I I}(z)$. The reionization redshift $z_{r}$ can approximately determined by requiring $Q_{H I I}\left(z_{r}\right) \geq 1$. The equation of $Q_{H I I}(z)$ is given by (e.g. Barkana \& Loeb 2001)

$$
\frac{d Q_{H I I}}{d t}=\frac{N_{i o n}(t)}{0.76} \frac{d M(>\rho / \bar{\rho})}{d t}-\frac{1}{a^{3}} \alpha_{B} C_{H I I}(t) \bar{n}_{H}^{0} Q_{H I I},
$$

where the factor 0.76 is from the fraction of hydrogen in the cosmic baryons, $\bar{n}_{H}^{0}=1.88 \times 10^{-7}\left(\Omega_{b} \mathrm{~h}^{2} / 0.022\right) \mathrm{cm}^{-3}$ is the present value of the mean number density of hydrogen, $N_{i o n}(t)$ is the mean ionization photons produced by each baryon in collapsed objects and might be $t$-dependent. The first term on the r.h.s. of eq.(12) accounts for the ionizing source. The ionization photons produced by primordial black holes are negligible because of the spectral index $n \leq 1$ on small scales (He \& Fang, 2002). The second term on the r.h.s. of eq.(12) is from the recombination, in which $a$ is the cosmic factor, $\alpha_{B}$ is the case $\mathrm{B}$ recombination coefficient $\simeq 2.6 \times 10^{-13} \mathrm{~cm}^{3} \mathrm{~s}^{-1}$ for hydrogen at $T=10^{4} \mathrm{~K}$, and $C_{H I I}(t)$ is the clumping factor of $H I I$.

Solving eq.(12) yields

$$
Q_{H I I}(t)=\int_{0}^{t} \frac{N_{i o n}(t)}{0.76} \frac{d M(>\rho / \bar{\rho})}{d t^{\prime}} e^{F\left(t^{\prime}, t\right)} d t^{\prime}
$$

where

$$
F\left(t^{\prime}, t\right)=-\alpha_{B} \bar{n}_{H}^{0} \int_{t^{\prime}}^{t} \frac{C_{H I I}\left(t^{\prime \prime}\right)}{a^{3}\left(t^{\prime \prime}\right)} d t^{\prime \prime}
$$

In the solution (13), an uncertain parameter is $N_{i o n}$, which depends on the atomic processes in star formation. As usual, one can express this number by $N_{\text {ion }}=N_{\gamma} \times f_{\text {star }} \times f_{\text {esc }}$, where 
$f_{\text {star }}$ - the fraction of collapsed baryon that formed star; $N_{\gamma}$ - the ionization photon number produced per baryon in star; and $f_{e s c}$ - the fraction of photon that escaped from galaxy. Generally, the first generation stars are massive (Abel et al. 2002, Bromm et al. 2002), and their lifetime are much less than the Hubble time at high $\mathrm{z}$ that we are interested. Thus, it would be reasonable to approximate the photons $N_{\gamma}$ being produced at the same redshift as collapsed clouds.

The number $N_{\gamma}$ is quite different for stars with different mass and metallicity. With the IMF of Scalo (1998), one has $N_{\gamma} \approx 4000$ for stars with metallicity $\sim 0.05 Z_{\odot}$. For the Salpeter's IMF, $N_{\gamma}$ is $\sim 6000$ in average for stars with masses of 1 to $100 \mathrm{M}_{\odot}$. For stars with zero metallicity, the number $N_{\gamma}$ could be as high as $10^{5}$ (Bromm et al. 2001, Venkatesan et al. 2003). Observational estimations of $f_{\text {star }}$ and $f_{\text {esc }}$ generally yield $f_{\text {star }} f_{\text {esc }} \sim 10^{-2}$. To sketch the reionization history driven by the mechanism discussed in $\S 3.1$, we use a simplest model of $N_{i o n}$, i.e. let $N_{\text {ion }}$ to be constant ( $z$-independent), and takes the value of 40,60 , and 80 respectively, which covers the possible range of $N_{\text {ion }}$.

Figure 4 plots the filling factor $Q_{H I I}$ vs. redshift $z$ in the three cosmological models with $N_{i o n}=40$, in which we take $C_{H I I}(z)=C(z)$. The curve of the model WMAP2 is similar to the COBE, while the WMAP1 shows a lower filling factor. This is because both the spectral index $n_{s}$ and the normalization of power spectrum $\sigma_{8}$ of the WMAP1 are smaller than other two models, and accordingly, the WMAP1 gives smaller variance $\sigma_{0}$ on the Jeans length of the IGM. From Fig. 4, the redshift of reionization is in the range $18>z_{r}>16$.

We also estimate $z_{r}$ by taking $N_{\text {ion }}=40,60$, and 80 , and $\rho / \bar{\rho}=6,8,10$. The results are tabulated in Table 2. In all the considered parameter-range, the redshift of reionization $z_{r}$ is $>10$. This result is consistent with the BFFJ, which also found $z_{r}>10$ but using different method. The BFFJ's conclusion is based on the evolution of the mass density and 
Table 2: Reionization redshift $\left(z_{r}\right)$

\begin{tabular}{l|lllll}
\hline$\rho / \bar{\rho}$ & 6 & 6 & 6 & 8 & 10 \\
\hline$N_{\text {ion }}$ & 40 & 60 & 80 & 40 & 40 \\
\hline WMAP1 & 15.8 & 17.0 & 17.4 & 14.0 & 12.9 \\
WMAP2 & 18.0 & 19.5 & 20.4 & 16.0 & 14.9 \\
COBE & 17.8 & 19.0 & 20.0 & 15.9 & 14.6 \\
\hline
\end{tabular}

velocity profiles of hydrogen clouds. Clearly, both approaches arrives at the same result that the reionization could occur much earlier than the redshift of an appearance of the complete Gunn-Peterson trough of Ly $\alpha$ absorption.

The assumption of $C_{H I I}(z)=C(z)$ is correct only if the density distribution of $H I I$ follows the distribution of total IGM. Since HII may favor to locate nearby collapsed clouds, we should use a biased relation $C_{H I I}(z)=b C(z)$. The bias parameter $b$ can be estimated by

$$
b=\left[\frac{\overline{\rho^{4}}}{{\overline{\rho^{2}}}^{2}}\right]^{1 / 2}=e^{2 \sigma_{0}^{2}},
$$

where we used eq.(6). Since $0.4<\sigma_{0}^{2}<0.8$ (Fig. 1), we have $2<b<5$. These values of $b$ is consistent with the estimation by the property of collapsed clouds (Mo \& White 1996, Fang \& Jing 1998). Because the velocity dispersion of collapsed clouds is small [Eq.(10)], the parameter $b$ should be in the above-mentioned range. Figure 5 plots the filling factor $Q_{H I I}$ vs. redshift $z$ for the model $W M A P 2, \rho / \bar{\rho}=6$ and $N_{i o n}=40$, but taking the biased clumping factor of $H I I$ with parameter $b=1.5,2$ and 4 . The results show that the uncertainty given by the bias factor is about the same as the uncertainty given by cosmological parameters (Fig. 4). Therefore this uncertainty will not affect the conclusions shown below. 


\subsection{The Redshift of the Gunn-Peterson Transparency}

Although $Q_{H I I}$ is $\simeq 1$ at $z_{r}$, it does not mean that $Q_{H I I} \simeq 1$ for all $z<z_{r}$, because $\sigma_{0}$ fell at $z=z_{r}$. For a self-consistent calculation of $Q_{H I I}$ in the epoch of $z<z_{r}$, we should use the lower branch of $\sigma_{0}$ as shown in Fig. 1.

The filling factor $Q_{H I I}$ at $z<z_{r}$ can also be calculated using equation (12). Figure 6 plots the results of $Q_{H I I}$ vs. redshift in the model WMAP2 with the parameters $\left(\rho / \bar{\rho}, N_{\text {ion }}\right)$ of $(6,40),(6,80)$, and $(8,40)$. Other models yield similar curves of $Q_{H I I}$. A common feature is that the reionization $Q_{H I I}$ drops to a level less than 1 after $z_{r}$, and then keeps $\simeq 0.5$ in the epoch of redshift $z_{r}>z>z_{g p}$. It is due to the decrease of the star formation rate when $\sigma_{0}$ drops back to $\sim 0.4$. When $z \rightarrow z_{g p}, \sigma_{0}$ becomes higher again [Fig. 1], and therefore, $Q_{H I I}$ quickly approaches to 1.

Each curves of Fig. 6 show that there are two solutions to the equation $Q_{H I I}(z) \simeq 1$, the first is at $z_{r}>15$, and the second is at $z_{g p} \sim 6-8$. This corresponds to the two phases of the star formation. During the period between the redshift $z_{r}$ and $z_{g p}$, the IGM is in multi-phase. The IGM consists of neutral and ionized hydrogen, HI and HII. Both components are comparable, and opaque for Ly $\alpha$ photons. However, the temperature of such multiphase IGM can be of the order of $10^{4} \mathrm{~K}$ in average. Therefore, it is self-consistent to apply the bottom branch of the $\sigma_{0}$ of Fig. 1 .

When $z \rightarrow z_{g p}$, the filling factor of neutral hydrogen becomes small quickly. The IGM is then transparent for Ly $\alpha$ photons when the volume filling factor of HI is less than $10^{-4}$, and thus the redshift $z_{g p}$ corresponds to the onset of the Gunn-Peterson transparency.

It should be pointed out that the two peak curve of $Q_{H I I}$ actually depends on the sharply dropping of $\sigma_{0}$ at $z_{r}$ (Fig. 1). As has been discussed in $§ 3.1$, more realistic model should replace the sudden increase of IGM temperature by a soft process. In this case, $Q_{H I I}$ 
probably never reached 1 at $z_{r}$, but at most shows a peak around $z_{r}$. Therefore, before $z_{g p}$, the IGM has never been ionized to the level of $1-Q_{H I I}<10^{-4}$. Thus, the redshift $z_{g p}$ is the first time of the IGM becoming the Gunn-Peterson transparency. Table 3 gives $z_{g p}$ for three sets of parameters.

Figure 6 and Table 3 shows that the redshift interval $z_{r}-z_{g p}$ is always as large as $\simeq 10-12$, which is not sensitive to the adopted parameters. This is because long duration from $z_{r}$ to $z_{g p}$ is essentially due to the zigzag $z$-dependence of the $\sigma_{0}$, which is less-sensitive to cosmological parameters, and the details of start formation. The temperature of the IGM increases by a factor of about $10^{4}$ during the reionization, while the entropy parameter

$T / n_{H}^{2 / 3}$ also increases by few orders. Since high temperature and high entropy prevent baryonic gas from collapsing into the gravity well of dark matter, the reionization is slowed down with the increase of temperature and entropy of the IGM. It behaves like a negative feedback mechanism: once the rate of star formation is higher, the UV background produced by the star formation is also higher, and then more baryonic gas will be heated to higher temperature and higher entropy state. This finally yields a suppression on the rate of star formation. Therefore, the long duration of reionization is mainly given by the evolution of the temperature and entropy of the baryonic gas during the reionization.

\subsection{Optical Depth to the CMB}

If the reionization lasts the long period of $z_{r}-z_{g p}$, the number density of free electrons is significant through the whole epoch $z_{r}>z>z_{g p}$. One can then expect that in this reionization history the electron scattering optical depth to the CMB would be much larger than models with $z_{r} \simeq z_{g p}$. With the solution of $Q_{H I I}$, the electron scattering optical depth 
to the CMB can be calculated by (e.g. Hui et al. 2003)

$$
\tau_{e}=0.0525 \times \Omega_{b} h \int_{0}^{z_{r}} \frac{V_{H I I}(z)(1+z)^{2} d z}{\sqrt{\Omega_{m}(1+z)^{3}+\Omega_{\Lambda}}},
$$

where $V_{H I I}(z)$ is the volume filling factor of $H I I$. Eq.(16) considered only the electrons from ionized hydrogen. To consider the contribution of He, we had assumed the filling factor of HeII to be identical to HII, i.e. the optical depth is increased by a factor of 1.08. The HeII $\rightarrow$ HeIII ionization occurred at redshift $\simeq 3.3$ (Theuns et al. 2002), and therefore, the correction of HeIII on $\tau_{e}$ is no more than 0.001 .

The relation between the volume filling factor $V_{H I I}$ and $Q_{H I I}$ is dependent on the statistical behavior of the formation of the ionized region. If the ionized hydrogen region increase, $d Q_{H I I}(t)$ in the duration $t$ to $t+d t$ is without spatial memory, the formation probability would be uniform in space regardless whether there are ionized region formed at time before $t$. Thus, we have equation $d V_{H I I} / d t=\left(1-V_{H I I}\right) d Q_{H I I} / d t$, and the solution is $V_{H I I}(z)=1-\exp \left[-Q_{H I I}(z)\right]$. However, the formation of the ionized region probably is not without spatial memory. The probability of forming collapsed clouds at the position where already hosted collapsed clouds should be low. Therefore, the process of the ionized hydrogen region formation most likely is self-avoid, i.e. the process has less probability at the positions where the process has been visited(e.g. Cardy 1996). For a perfectly self-avoiding process, no position can host the formation process more than once (e.g. Cardy 1996). In this case, we have $V_{H I I}(z)=Q_{H I I}(z)$ till $Q_{H I I} \leq 1$.

Table 3 lists the results of $z_{r}, z_{g p}$ and $\tau_{e}$ for the model WMAP2 with parameters $\left(N_{\text {ion }}, \rho / \bar{\rho}\right)$ specified by $(6,40),(8,40)$ and $(6,80)$. The optical depth $\tau_{e}(1)$ is for the model without any self-avoiding, while $\tau_{e}(2)$ is for model with perfectly self-avoiding. Real number probably is in between of these two ultimate models. All results shown in Table 3 give a low $z_{g p} \sim 6-8$ and a high $\tau_{e} \sim 0.12-0.19$. These results are excellently consistent with the observations of the redshift of the Gunn-Peterson trough and the electron optical depth 
Table 3: Reionization redshift, Gunn-Peterson redshift and optical depth to CMB

\begin{tabular}{ll|llll}
\hline$\rho / \bar{\rho}$ & $N_{\text {ion }}$ & $z_{r}$ & $z_{g p}$ & $\tau_{e}(1)$ & $\tau_{e}(2)$ \\
\hline 6 & 40 & 18.0 & 7.0 & 0.150 & 0.181 \\
8 & 40 & 16.0 & 6.0 & 0.123 & 0.154 \\
6 & 80 & 20.4 & 7.9 & 0.159 & 0.189 \\
\hline
\end{tabular}

to the CMB. The key of reconciling the two observation is that there exists a long period of the universe to have $0.1<V_{H I I}<1-10^{-4}$. The free electrons in this period contribute to a high value of $\tau_{e}$. On the other hand, the Gunn-Peterson transparency occurred at the end of this epoch, and the redshift $z_{g p}$ is small.

\section{Discussion and Conclusions}

We showed with the LN model that the reionization and Gunn-Peterson transparency emerged at very different time. The thermal history of the universe around reionization can roughly be divided into three epochs: 1.) cold dark age $z>z_{r}$, in which baryonic gas is cold, and opaque for Ly $\alpha$ photons; 2.) hot dark age $z_{r}>z>z_{g p}$, in which a substantial part of baryon gas is ionized and hot, but it is still opaque for Ly $\alpha$ photons, 3.) bright age $z<z_{g p}$. For the LCDM models favored by the WMAP, we have $z_{r} \simeq 13-19$ and $z_{g p} \simeq 6-8$. That is, the hot dark age is in the redshift interval from 13-19 to 6 -8. This thermal history naturally yields a high optical depth $\tau_{e}=0.12-0.19$ and a low $z_{g p}=6-8$, which is in excellent agreement with the observations of $\tau_{e}$ (WMAP) and $z_{g p}$ (Gunn-Peterson trough).

The long duration from $z_{r}$ to $z_{g p}$ is essentially due to the evolution of the temperature and entropy of the baryonic gas during the reionization. For all the considered cosmological models, there is always a drop-off of $\sigma_{0}$ at $z_{r}$. Therefore, a large difference $z_{r}-z_{g p}$ is a 
common property of these models. The result of low $z_{g p}$ and high $\tau_{e}$ is less sensitive to the cosmological parameters.

This result is also weakly dependent on the details of production of ionization photons from stars. This is due to the feedback mechanism of the start formation (§3.3). The higher rate of ionization photon production will cause a stronger input of entropy to baryonic gas. It then leads to a lower rate of the collapsing of baryonic clouds, suppressing the rate of star formation and the UV photon production. Therefore, model, which properly considers the dramatic evolution of the temperature and entropy of the IGM, will generally yield a low $z_{g p}$ and a high $\tau_{e}$.

The LN model provides an intuitive and quantitative description of the effect of the IGM temperature evolution upon the reionization history. This is because the LN model use directly variance $\sigma_{0}$ as a key parameter. It has already been recognized that the variance $\sigma_{0}$ is more effective and successful in modeling the IGM detected by QSO's Ly $\alpha$ forests (Bi \& Davidsen 1997). We showed in this paper that the LN model provides a plausible description of photoionized and neutral IGM not only in the redshift range $z \leq 4$, but extended to $z \simeq 15$.

The hot dark age between the redshifts $z_{r}$ and $z_{g p}$ is an interesting issue. During that period, star formation is significant, although the rate of star formation is lower than that at $z_{r}$. On the other hand, baryonic gas is in multiphase, and consists of comparable components of HI and HII. The gas has been already hot. Therefore, it is expected to find some observable signatures, such as high redshift $21 \mathrm{~cm}$ emission, of the hot dark age.

JRL thanks Mr. Guopeng Shen and Jun Yu for useful discussions. LLF acknowledges support from the National Science Foundation of China (NSFC) and National Key Basic Research Science Foundation. 


\section{REFERENCES}

Abel, T., Bryan, G.L., \& Norman, M.L., 2002, Science, 295, 93

Avelino, P.P., \& de Carvalho J.P.M., 1999, MNRAS, 310, 1170

Barkana, R., \& Loeb, A., 2001, Phys. Rep., 349, 125

Bi, H.G. 1993, ApJ, 405, 479

Bi, H.G \& Davidsen, A.F., 1997, ApJ, 479, 523.

Bi, H.G., \& Fang, L.Z., \& Feng, L.L. \& Jing, Y.P. 2003, ApJ, 598, 1

Bromm, V., Coppi, P.S., \& Larson, R.B., 2002, ApJ, 564, 23

Bromm, V., Kudritzki, R.P., \& Loeb, A., 2001, ApJ, 552, 464

Cardy, J. 1996, Scaling and renormalization in statistical physics, (Cambridge)

Carroll, S.M., Press, W.H., \& Turner, E.L., 1992, ARA\&A, 30, 499

Cen, R., 2003, ApJ, 591, 12

Ciardi, B., Ferrara, A., \& White, S.D.M., 2003, MNRAS, 344, 7

Eisenstein, D. \& Hu, W., 1999, ApJ, 511, 5

Fan, X., et al., 2002, AJ, 123, 1247

Fang, L.Z. \& Jing, Y.P. 1998, ApJ502, L95

Feng L.L. \& Fang, L.Z. 2000, ApJ, 535, 519

Feng, L.L., Pando, J., \& Fang, L.Z. 2003, ApJ, 587, 487

Gnedin, N. \& Hui, L. 1998, MNRAS, 296, 44

Haiman, Z., \& Holder, G.P., 2003, astro-ph/0302403

He, P. \& Fang, L.Z. 2002, ApJ, 568, L1.

Holder, G., Haiman, Z., Kaplinghat, M., \& Knox, L., 2003, ApJ, in press (astro-ph/0302404) 
Hui L. \& Haiman Z., 2003, astro-ph/0302439

Jamkhedkar, P., Feng, L.L., Zheng, W., Kirkman, D., Tytler, D. \& Fang, L.Z. 2003, MNRAS, 343, 1110J

Jamkhedkar, P., Zhan, H. \& Fang, L.Z. 2000, ApJ, 543, L1

Jing, Y.P., \& Fang, L.Z., 1994, ApJ, 432, 438

Jones, B.T., 1999, MNRAS, 307, 376

Kogut, A., et al., 2003, ApJS, 148, 161

Lahav, O., Rees, M.J., Lilje, P.B., \& Primack, J.R., 1991, MNRAS, 251, 128

Medvigy, D., \& Loeb, A., 2001, submitted to ApJ, (astro-ph/0110014)

Matarrese, S. \& Mohayaee, R., 2002, MNRAS, 329, 37

Mo, H.J. \& White, S.D.M. 1996, MNRAS, 282, 347

Ostriker, J.P. \& Gnedin, N.Y., 1996, ApJ, 472, L63

Onken, C.A. \& Miralda-Escudé 2003, astro-ph/0307184

Sarazin, C.L., \& Bahcall, J.N., 1977, ApJS, 34, 451

Scalo, J., 1998 in Asp conference series Vol. 142, The Stellar Initial Mass Function, eds. G. Gilmore \& D. Howell, p.201

Spergel, D.N., et al., 2003, ApJS, 175

Theuns, T., Bernardi, M., Frieman, J., Hewett, P., Schaye, J., Sheth, R. K., \& Subburao, M., 2002, ApJ, 574, 111

Vanmarcke, E., 1983, Random Field, (MIT, Mass)

Venkatesan, A., Tumlinson, J., \& Shull, J.M., 2003, ApJ, 584, 621

Wyithe, S., \& Loeb, A., 2003, ApJ, 588, 69 
Zel'dovich, Ya.B., Ruzmaikin, A.A., \& Sokoloff, D.D., 1990, The Almighty Chance, p. 190, (World Scientific, Singapore). 


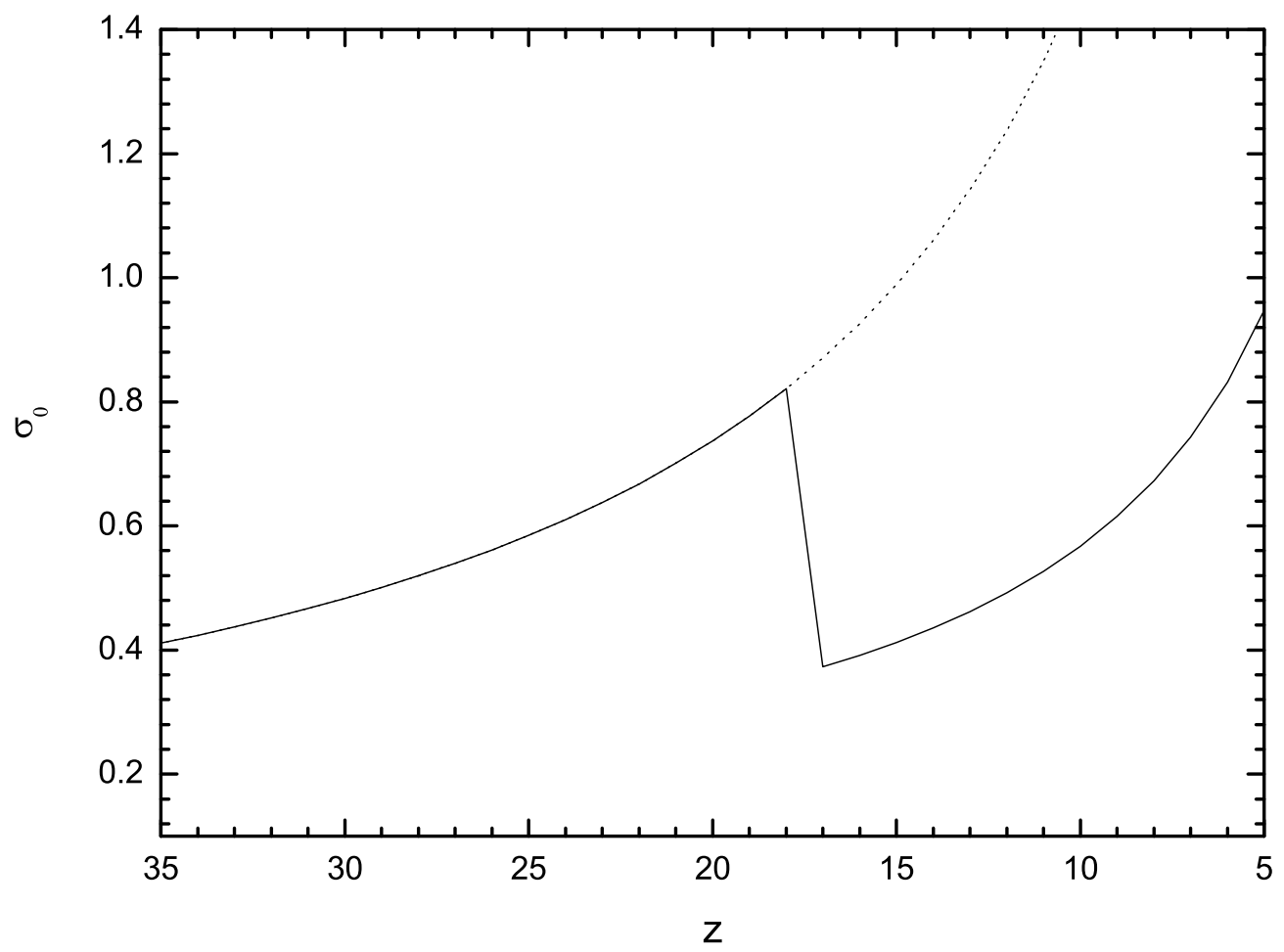

Fig. 1.- The variances of the linear density fluctuations at the Jeans length as a function of redshift for model WMAP2. The reionization redshift $z_{r}$ is taken to be 17 . The dot line at $z<z_{r}$ is for the variances without considering the reionization. The solid line at $z<z_{r}$ is considered the dropping of $\sigma_{0}$ at $z_{r}$ due to the increase of the temperature of IGM. 


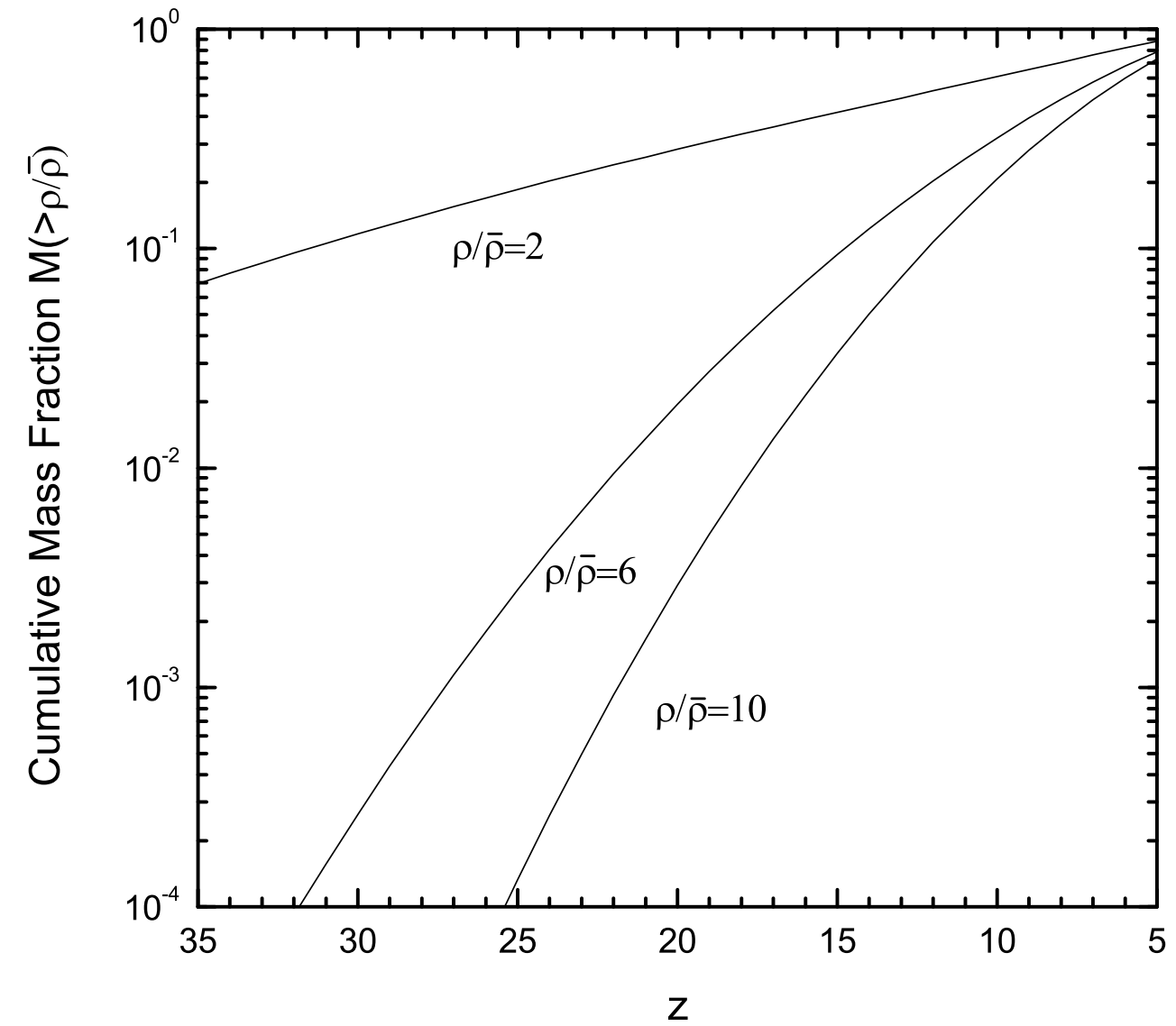

Fig. 2.- The cumulative mass fraction $M(>\rho / \bar{\rho})$ for model WMAP2. $\rho / \bar{\rho}$ is taken to be 2, 6 and 10. At $z<z_{r}$, the $\sigma_{0}$ is taken from the dot line in Fig. 1. 


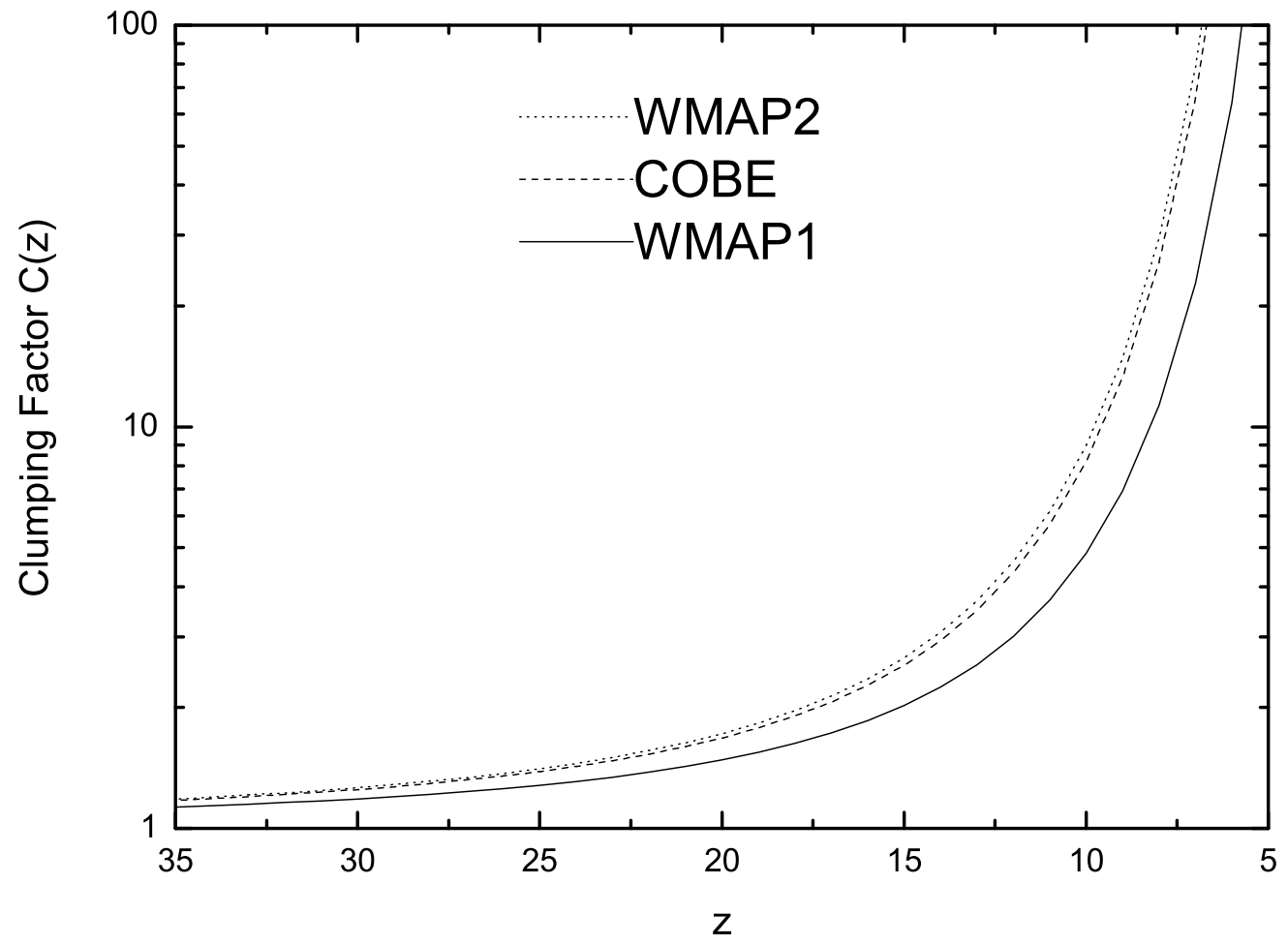

Fig. 3.- The clumping factor $\mathrm{C}(\mathrm{z})$ for the three cosmological models given in Table 1 . 


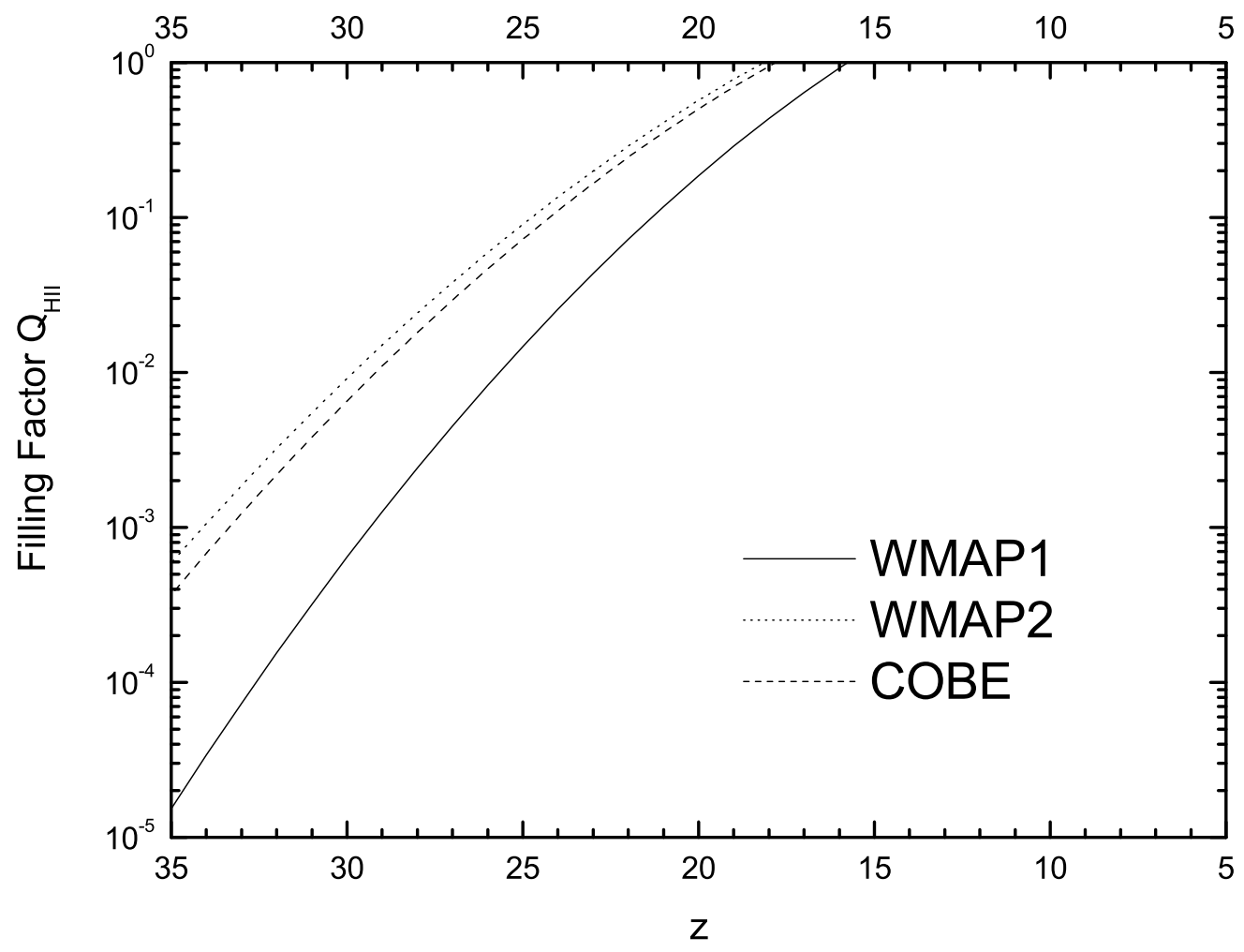

Fig. 4.- The filling factor $Q_{H I I}$ vs. redshift of the three cosmological models, WMAP1, WMAP2 and COBE. $\rho / \bar{\rho}$ and $N_{\text {ion }}$ are taken to be 6 and 40 , respectively. 


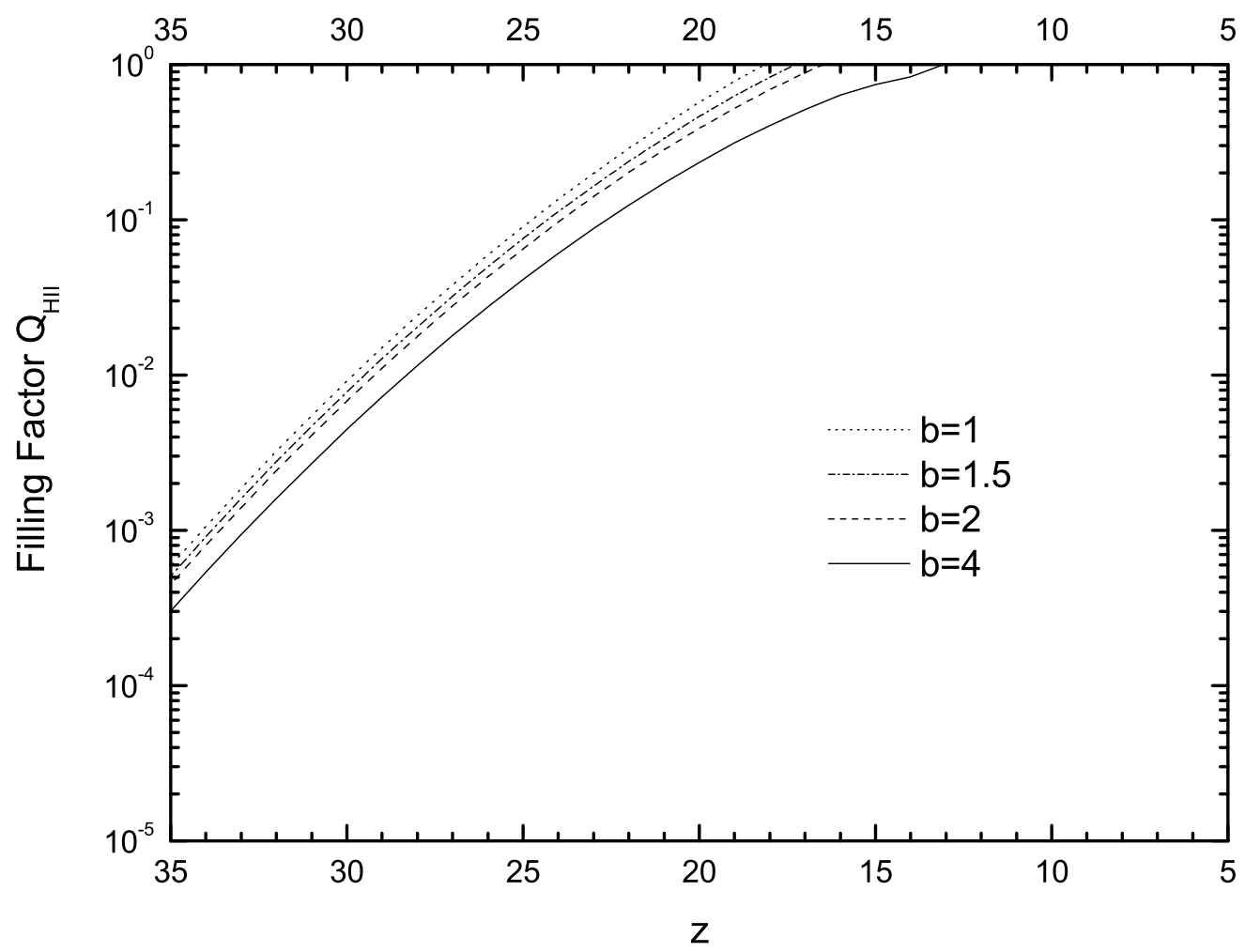

Fig. 5.- The filling factor $Q_{H I I}$ vs. redshift of the model WMAP2, $\rho / \bar{\rho}=6$ and $N_{\text {ion }}=40$. The bias parameter $b$ of the clumping factor $C_{H I I}=b C$ is taken to be $1.5,2$ and 4 . 


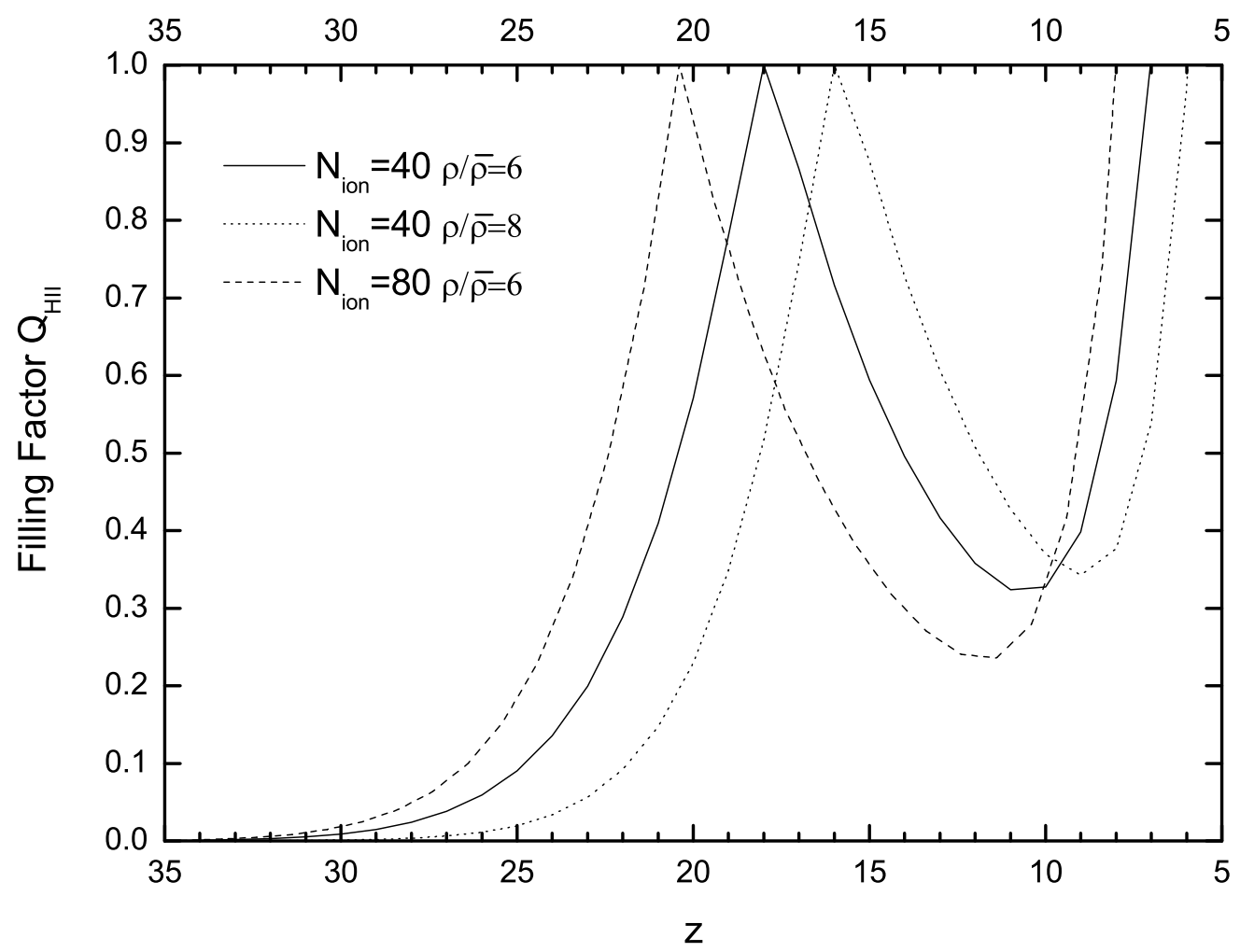

Fig. 6.- The redshift evolution of the volume filling factor $Q_{H I I}(\mathrm{Z})$ from $z>z_{r}$ to $z_{g p}$ for model WMAP2. $\rho / \bar{\rho}$ and $N_{\text {ion }}$ are taken to be $(8,40)$ (dot), $(6,40)$ (solid), and $(6,80)$ (dash). The reionization redshifts $z_{r}$ is given by $Q_{H I I} \approx 1$ at $z>10$. The redshift of the Gunn-Peterson transparency $z_{g p}$ is given by $Q_{H I I} \approx 1$ at $z<10$. 